\title{
Fermented green tea extract exhibits hypolipidaemic effects through the inhibition of pancreatic lipase and promotion of energy expenditure
}

\author{
Dae-Bang Seo ${ }^{1} \dagger$, Hyun Woo Jeong ${ }^{2} \dagger$, Yeon-Ji Kim ${ }^{1} \dagger$, Sukyung Kim ${ }^{2}$, Jeongkee Kim ${ }^{2}$, Ji Hae Lee ${ }^{3}$, \\ Kyungmi Joo ${ }^{4}$, Jin Kyu Choi ${ }^{5}$, Song Seok Shin ${ }^{2 *}$ and Sung-Joon Lee ${ }^{1_{*}}$ \\ ${ }^{1}$ Department of Biotechnology, BK21-PLUS, College of Life Sciences E Biotechnology, Korea University, Seoul O2841, \\ Republic of Korea \\ ${ }^{2}$ Beauty Food Division, Amorepacific Corporation RED Center, Gyeonggi-Do 17074, Republic of Korea \\ ${ }^{3}$ Medical Beauty Division, Amorepacific Corporation RED Center, Gyeonggi-Do 17074, Republic of Korea \\ ${ }^{4}$ Skin Research Division, Amorepacific Corporation RED Center, Gyeonggi-Do 17074, Republic of Korea \\ ${ }^{5}$ QC team, Aeustra Corporation, Gyeonggi-Do 17573, Republic of Korea \\ (Submitted 17 May 2016 - Final revision received 7 December 2016 - Accepted 19 December 2016 - First published online 30 January 2017)
}

\section{Abstract}

Hyperlipidaemia is a major cause of atherosclerosis and related CVD and can be prevented with natural substances. Previously, we reported that a novel Bacillus-fermented green tea (FGT) exerts anti-obesity and hypolipidaemic effects. This study further investigated the hypotriglyceridaemic and anti-obesogenic effects of FGT and its underlying mechanisms. FGT effectively inhibited pancreatic lipase activity in vitro $\left(\mathrm{IC}_{50}, 0.48 \mathrm{mg} / \mathrm{ml}\right)$ and ameliorated postprandial lipaemia in rats (26\% reduction with $500 \mathrm{mg} / \mathrm{kg}$ FGT). In hypertriglyceridaemic hamsters, FGT administration significantly reduced plasma TAG levels. In mice, FGT administration ( $500 \mathrm{mg} / \mathrm{kg}$ ) for 2 weeks augmented energy expenditure by $22 \%$ through the induction of plasma serotonin, a neurotransmitter that modulates energy expenditure and mRNA expressions of lipid metabolism genes in peripheral tissues. Analysis of the gut microbiota showed that FGT reduced the proportion of the phylum Firmicutes in hamsters, which could further contribute to its anti-obesity effects. Collectively, these data demonstrate that FGT decreases plasma TAG levels via multiple mechanisms including inhibition of pancreatic lipase, augmentation of energy expenditure, induction of serotonin secretion and alteration of gut microbiota. These results suggest that FGT may be a useful natural agent for preventing hypertriglyceridaemia and obesity.

Key words: Fermented green tea: Pancreatic lipase: Serotonin: Hyperlipidaemia: Energy expenditure: Gut microbiota

Energy homoeostasis is tightly regulated by the interaction of central and peripheral organs that control total energy intake and energy expenditure. Positive energy balance leads to excess storage of energy molecules, primarily in the form of TAG, in metabolic tissues including adipose, liver, muscle and pancreatic tissues, causing cellular dysfunction and lipotoxicity $^{(1,2)}$, which are the major causes for developing metabolic disorders such as type 2 diabetes and related $\mathrm{CVD}^{(3-7)}$. Accordingly, treatment and prevention of hyperlipidaemia is critical for lowering the risk of $\mathrm{CVD}^{(8)}$

Several effective and potent hypolipidaemic drugs are available including statins, fibrates and metformin ${ }^{(9-12)}$; however, these drugs may not be tolerated for long-term treatment and may cause significant side-effects. Thus, natural substances have been considered alternatives for the prevention of dyslipidaemia in humans - for example, resveratrol and berberine ameliorate hyperlipidaemia and related metabolic disorders by activating AMP-activated protein kinase (AMPK) ${ }^{(13-15)}$

On the other hand, numerous animal studies have demonstrated that green tea and its processed products (e.g. oolong tea and black tea) exhibit lipid-lowering effects ${ }^{(16-25)}$. The hypotriglyceridaemic effects of green tea and its derivatives have also been well documented in clinical trials and have recently been intensively reviewed ${ }^{(26)}$. For instance, consumption of green tea inhibits lipid digestion and absorption after a meal ${ }^{(27)}$, and long-term supplementation with green tea improves plasma lipid profiles and increases the levels of antioxidants $^{(28,29)}$. Black tea also exerts hypotriglyceridaemic effects in humans ${ }^{(30,31)}$. A meta-analysis of human studies revealed that black tea reduces serum cholesterol and LDL concentrations $^{(32)}$. Similar to other hypotriglyceridaemic agents (e.g. metformin and berberine), green and black teas activate

Abbreviations: AMPK, AMP-activated protein kinase; FF, fenofibrate; FGT, fermented green tea; HFD, high-fat diet; LPL, lipoprotein lipase.

* Corresponding authors: S.-J. Lee, fax +82 23290 3653, email junelee@korea.ac.kr; S. S. Shin, fax +82 312818392 , email ssshin@amorepacific.com

$\dagger$ These authors contributed equally. 
AMPK and inhibit 3-hydroxy-3-methyl-glutaryl-CoA reductase ${ }^{(33)}$ - key molecules involved in the control of lipid metabolism. In addition, in humans, oolong tea enhances lipid excretion in faeces ${ }^{(34)}$. Collectively, these data suggest that green tea and processed green teas may be effective agents for improving hyperlipidaemia and its related metabolic complications.

In a previous study, we proposed that fermented green tea (FGT) with Bacillus sp. had anti-obesogenic effects in dietinduced obese mice ${ }^{(35)}$. We observed that FGT reduced plasma lipid levels as well as plasma glucose levels, implying that, similar to green tea and related products, FGT exerts hypotriglyceridaemic effects. To elucidate the effects and the underlying mechanism through which FGT influences lipid metabolism, we designed additional experiments in this study. Specifically, we examined the hypotriglyceridaemic effects of FGT in acute and diet-induced chronic hyperlipidaemic animal models. To determine the molecular mechanisms of FGT-mediated hypotriglyceridaemic effects, we evaluated the enzymatic activity of pancreatic lipase. We also measured energy expenditure and the expressions of lipid metabolism-related genes in FGT-administered animals. Finally, we analysed gut microbiota from faecal samples.

\section{Methods}

\section{Reagents and fermented green tea extract preparation}

Triton WR-1339 (Triton, a lipoprotein lipase (LPL) inhibitor) and fenofibrate (FF, PPAR $\alpha$ agonist) were purchased from Sigma. FGT extracts were produced by Mizon Co., as described in the previous study ${ }^{(35)}$ with the de-caffeination method. In brief, dried green tea leaves were mixed with $1 \%$ sucrose and Bacillus subtilis $\left(5 \times 10^{7}\right.$ colony-forming unit) and fermented at $50^{\circ} \mathrm{C}$ for $3 \mathrm{~d}$, followed by further incubation at $90^{\circ} \mathrm{C}$ for $4 \mathrm{~d}$ to remove remaining $B$. subtilis. After fermentation, the FGT was dried and extracted with $50 \%$ ethanol at $70^{\circ} \mathrm{C}$ for $2 \mathrm{~h}$. Analysis of catechin and caffeine composition was performed as described previously ${ }^{(35)}$. The composition of catechins and caffeine in the FGT is shown in Table 1.

\section{Acute hypotriglyceridaemic effect of fermented green tea}

All animal experiments were approved by the Amorepacific Institutional Animal Care and Use Committee (PQ13-S007) and

Table 1. Composition of catechins $(C)$ in green tea and fermented green tea (FGT)

\begin{tabular}{lcc}
\hline Components & Green tea $(\% \mathrm{w} / \mathrm{w})$ & FGT $(\% \mathrm{w} / \mathrm{w})$ \\
\hline C & 26.56 & 8.27 \\
GC & 1.52 & 1.26 \\
EGC & 11.7 & 1.32 \\
C & 0.39 & 0.22 \\
EC & 2.16 & 0.26 \\
EGCG & 8.74 & 2.63 \\
GCG & 0.82 & 2.02 \\
ECG & 1.23 & 0.56 \\
Caffeine & 8.13 & 3.70 \\
Catechin + caffeine & 34.69 & 11.97 \\
\hline
\end{tabular}

GC, gallocatechin; EGC, epigallocatechin; EC, epicatechin; EGCG, epigallocatechin gallate; GCG, gallocatechin gallate; ECG, epicatechin gallate. adhere to the Organisation for Economic Cooperation and Development (OECD) guidelines. Sprague-Dawley (SD) male rats, 6-week-old, were purchased from the Central Laboratory Animal Inc. and maintained in a $12 \mathrm{~h}$ dark- $12 \mathrm{~h}$ light cycle chamber with controlled temperature of $22-25^{\circ} \mathrm{C}$ and $40-50 \%$ humidity. For adaptation, rats were fed normal chow ad libitum for 1 week. The average level of plasma TAG was not significantly different (online Supplementary Table S1). After adaptation, animals were divided into four groups (Saline, Triton, Triton + FGT and Triton + FF; $n$ 5/group). Triton was utilised to induce hyperlipidaemia, and FF was used as a positive control. Rats were orally injected with saline, $500 \mathrm{mg} / \mathrm{kg}$ of body weight of FGT or $65 \mathrm{mg} / \mathrm{kg}$ body weight of FF for $5 \mathrm{~d}$. After $5 \mathrm{~d}$, animals were fasted overnight, and the final administration of selected agents (saline, FGT and FF) was carried out $1 \mathrm{~h}$ before Triton treatment. Finally, Triton $(200 \mathrm{mg} / \mathrm{kg}$ body weight) was delivered to all rats except among those in the saline group through the tail vein. At 0, 3, 5, 18 and $20 \mathrm{~h}$ after Triton injection, blood samples were collected to measure plasma TAG levels. Plasma TAG levels were measured using an automated clinical chemistry analyzer (Cobas111; Roche).

\section{Prevention of diet-induced hyperlipidaemia by fermented green tea}

Golden Syrian male hamsters, 9-week-old male, purchased from the Central Laboratory Animal Inc., were maintained in a $12 \mathrm{~h}$ light $-12 \mathrm{~h}$ dark cycle chamber with controlled temperature of $21-25^{\circ} \mathrm{C}$ and $50-60 \%$ humidity. After being fed a commercial chow diet (Central Laboratory Animal Inc.) for 1 week, hamsters were fed a $45 \%$ high-fat diet (HFD) (Central Laboratory Animal Inc.) with $10 \%$ fructose in drinking water for 2 weeks, followed by a western diet (Central Laboratory Animal Inc.) with 10\% fructose in drinking water for another 2 weeks. At first, the hamsters were randomly assigned to four groups: the control (water as a vehicle and the western diet), FF (positive control; western diet with $100 \mathrm{mg} / \mathrm{kg}$ body weight of FF) and two FGT groups (200 and $400 \mathrm{mg} / \mathrm{kg}$ body weight of FGT with the western diet); diets were orally administered for 4 weeks. During the experiment, plasma samples were collected every 2 weeks, and the concentrations of TAG were analysed by a Cobas C111 automated clinical chemistry analyzer following the manufacturer's protocol. To examine the effect of long-term treatment with FGT on plasma TAG levels, 9-week-old male Golden Syrian hamsters were purchased from the Central Laboratory Animal Inc., adopted and fed a western diet as described above. Next, the hamsters were randomly assigned to five groups: control, FF (100 mg/kg body weight) and three FGT groups (200, 400 or $600 \mathrm{mg} / \mathrm{kg}$ body weight, respectively). Hamsters were administered water (as a vehicle), FGT or FF via oral gavage for 12 weeks, respectively. During administration of the reagent, a western diet with $10 \%$ fructose was still supplied to all hamsters. Plasma samples from hamsters were collected just before and after 12 weeks of treatment, and TAG concentrations were analysed as described above. All experiments involving mice and hamsters were performed according to a protocol approved by the Animal Experiment Committee of Korea University (Protocol No. KUIACUC-2013-139). 


\section{Pancreatic lipase activity assay}

Pancreatic lipase activity was measured as previously described $^{(36)}$. In brief, FGT was dissolved in distilled water (as a negative control) or in $50 \mu \mathrm{l}$ of 4-methylumbelliferyl oleate (4-MO; as a substrate; Sigma-Aldrich Co. LLC.) solution dissolved in an assay buffer (13 mm TRIS- $\mathrm{HCl}, 150 \mathrm{~mm} \mathrm{NaCl}$ and $1.3 \mathrm{~mm} \mathrm{CaCl}_{2}$ with $\left.\mathrm{pH} 8.0\right)$. Subsequently, $25 \mu \mathrm{l}$ of pancreatic lipase ( $50 \mathrm{U} / \mathrm{ml}$; Sigma-Aldrich Co. LLC.) was added and incubated at $25^{\circ} \mathrm{C}$ for $30 \mathrm{~min}$. To terminate the enzyme reaction, $100 \mu \mathrm{l}$ of sodium citrate $(100 \mathrm{mmol})$ was added to the reaction mixture. The amount of 4-methylumbelliferone released from 4-MO by pancreatic lipase was measured using TECAN M200 PRO fluorometric plate reader (TECAN Trading AG; excitation $355 \mathrm{~nm}$ and emission $460 \mathrm{~nm}$ ). The $\mathrm{IC}_{50}$ of FGT on pancreatic lipase was calculated from a regression line of the plots in the logarithm of FGT concentration $v$. pancreatic lipase activity graph.

\section{Measurement of energy expenditure and plasma} neurotransmitter levels

C57BL/6J male mice, 6-week-old, were purchased from the Central Laboratory Animal Inc. and maintained in a $12 \mathrm{~h}$ light$12 \mathrm{~h}$ dark cycle chamber with controlled temperature of $21-25^{\circ} \mathrm{C}$ and $50-60 \%$ humidity. For adaptation, mice were fed an AIN-76A diet (Central Laboratory Animal Inc.) ad libitum for 1 week. After adaptation, mice were fed an AIN-76A-based HFD ( $45 \%$ ) with orally administered $500 \mathrm{mg} / \mathrm{kg}$ body weight $/ \mathrm{d}$ of FGT. The same volume of distilled water was given to the control group for 2 weeks. $\mathrm{VO}_{2}$ and carbon dioxide production $\left(\mathrm{VCO}_{2}\right)$ were measured using the Oxylet Physiocage System (Panlab) and the software suite METABOLISM (version 2.2.01; Panlab). The respiratory exchange ratio used for estimating the RQ was calculated as $\mathrm{VCO}_{2}: \mathrm{VO}_{2}$, and energy expenditure was calculated according to the formula (kJ (kcal)/ $(\mathrm{d} \mathrm{kg} \cdot 0 \cdot 75))=\mathrm{VO}_{2} \cdot 1 \cdot 44 \cdot(3 \cdot 815+(1 \cdot 232 \cdot$ resting energy requirement $))$.

For neurotransmitter measurements, 6-week-old male C57/ BL6 mice were purchased from the Central Laboratory Animal Inc. and adapted for 1 week. After adaptation, mice were fed a $45 \%$ HFD. During the administration of a HFD, FGT $(500 \mathrm{mg} / \mathrm{kg}$ body weight) or water (as a vehicle) was orally administered for 8 weeks. After FGT administration, mice were fasted overnight, and blood samples were collected and centrifuged $\left(4^{\circ} \mathrm{C}\right.$, $3000 \mathrm{rpm}, 5 \mathrm{~min}$ ). Supernatants were transferred to new microcentrifuge tubes. Plasma levels of dopamine, norepinephrine and serotonin were measured using a dopamine ELISA kit (Abnova), norepinephrine ELISA kit (LifeSpan Biosciences) and serotonin ELISA kit (Abcam), respectively, following each manufacturer's instructions. White adipose tissue (WAT) and liver tissue were separated and stored at $-80^{\circ} \mathrm{C}$ for further use. All animal experiments were approved by the Amorepacific Institutional Animal Care and Use Committee (AP11-FR008) and adhered to the OECD guidelines.

\section{Pyrosequencing analysis of gut microbiota}

For pyrosequencing analysis, faeces samples were collected for 3 consecutive days before the animals were euthanised with $\mathrm{CO}_{2}$.
The stool samples were stored at $-80^{\circ} \mathrm{C}$ until analysis, and then genomic DNA was extracted from pooled faecal samples using the FastDNA ${ }^{\mathrm{TM}}$ SPIN kit for Faeces (MP Biomedical) according to the manufacturer's protocol. For pyrosequencing, amplification of genomic DNA was performed using barcoded primers that target the V1-V3 region of the bacterial 16S rRNA gene. Amplification, sequencing and basic analysis were performed according to the methods described by Chun et al. ${ }^{(37)}$ and were completed by ChunLab Inc. using the 454 GS FLX Titanium Sequencing Systems (Roche). Sequence reads were identified using EzTaxon-e database (http://eztaxon-e.ezbiocloud.net) ${ }^{(38)}$ on the basis of $16 \mathrm{~S}$ rRNA sequence data. We analysed the number of sequences, observed the diversity richness (operational taxonomic units (OTU)) and estimated the OTU richness (abundance-based coverage estimator and Chao1 indices). Bacterial community abundance and composition were generated using CLcommunity software (ChunLab Inc.).

\section{RNA isolation, complementary DNA synthesis and quantitative $R T-P C R$}

RNA from tissues was isolated using the RNeasy ${ }^{\circledR}$ Mini Kit (Qiagen) following the manufacturer's protocol. Each RNA sample $(2 \mu \mathrm{g})$ was subjected to complementary DNA (cDNA) synthesis using the RevertAid ${ }^{\mathrm{TM}}$ First Strand cDNA Synthesis Kit (Thermo Fisher Scientific). Relative mRNA levels were determined by quantitative RT-PCR (qRT-PCR) using the appropriate primers (Bioneer) as described previously ${ }^{(39)}$. Primer sequences used for qRT-PCR are provided in the online Supplementary Table S2.

\section{Activity of lipoprotein lipase}

HepG2 human hepatoma cells were obtained from the Korean Cell Line Bank and grown in Dulbeco's modified Eagle's medium (DMEM) supplemented 10\% FBS and 1\% penicillin and streptomycin (PEST) at $37^{\circ} \mathrm{C}$ in an atmosphere containing $5 \% \mathrm{CO}_{2}$. HepG2 were cultured in six-well plates at a density of $10^{6}$ cells/ well for $24 \mathrm{~h}$, and then cells were treated with various concentrations of FGT $(0 \cdot 03,0 \cdot 1,0 \cdot 3,1,3,10,30$ and $100 \mu \mathrm{g} / \mathrm{ml})$ in DMEM without FBS and PEST for another $24 \mathrm{~h}$. After incubation, the supernatants were collected, and human LPL concentrations were analysed using an ELISA-based Human LPL Assay Kit (Immuno-Biological Laboratories Co., Ltd), according to the manufacturer's instructions.

\section{Statistical analysis}

All data are shown as means with their standard errors. Student's $t$ test was performed for two-group comparison, and one-way ANOVA was performed for multiple-group comparison. $P<0.05$ was considered as significant.

\section{Results}

Fermented green tea relieves acute hyperlipidaemia in rats

In the previous study, we showed that FGT decreased plasma lipid levels in proportion to the reduction in body weight in 
diet-induced obese mice ${ }^{(35)}$. To elucidate whether FGT exerts hypotriglyceridaemic effects, regardless of body weight control, we administered FGT to SD rats by oral gavage for $5 \mathrm{~d}$. FGT administration did not significantly alter body weight (online Supplementary Table S3), implying that acute treatment of FGT is independent of body weight change with a normal diet. To provoke hyperlipidaemia acutely, we injected Triton, a LPL inhibitor, to SD rats. As shown in Fig. 1(a), Triton administration caused a robust increase in plasma TAG levels, whereas pretreatment with FF blunted the hyperlipidaemic effect induced by Triton. Similarly, FGT reduced hypertriglyceridaemia by $26 \%$ (Fig. 1(b)). Collectively, these results suggest that FGT exhibits hypotriglyceridaemic effects.

\section{Fermented green tea reduces plasma TAG levels in diet-induced hyperlipidaemic hamsters}

FGT partially improved acute hyperlipidaemia induced by Triton treatment (Fig. 1). To assess whether FGT inhibited dietinduced elevations in plasma lipid levels, we administered FGT (200 or $500 \mathrm{mg} / \mathrm{kg}$ body weight) to western diet-induced hyperlipidaemic hamsters for 4 weeks. Although low-dose FGT $(200 \mathrm{mg} / \mathrm{kg})$ failed to lower plasma TAG levels, FF $(100 \mathrm{mg} / \mathrm{kg})$ and high-dose FGT $(500 \mathrm{mg} / \mathrm{kg})$ treatment blunted further elevations in plasma TAG (Fig. 2(A)). These data imply that high doses of FGT are required to acutely lower plasma TAG levels. To further elucidate long-term and dose-responsive effects of
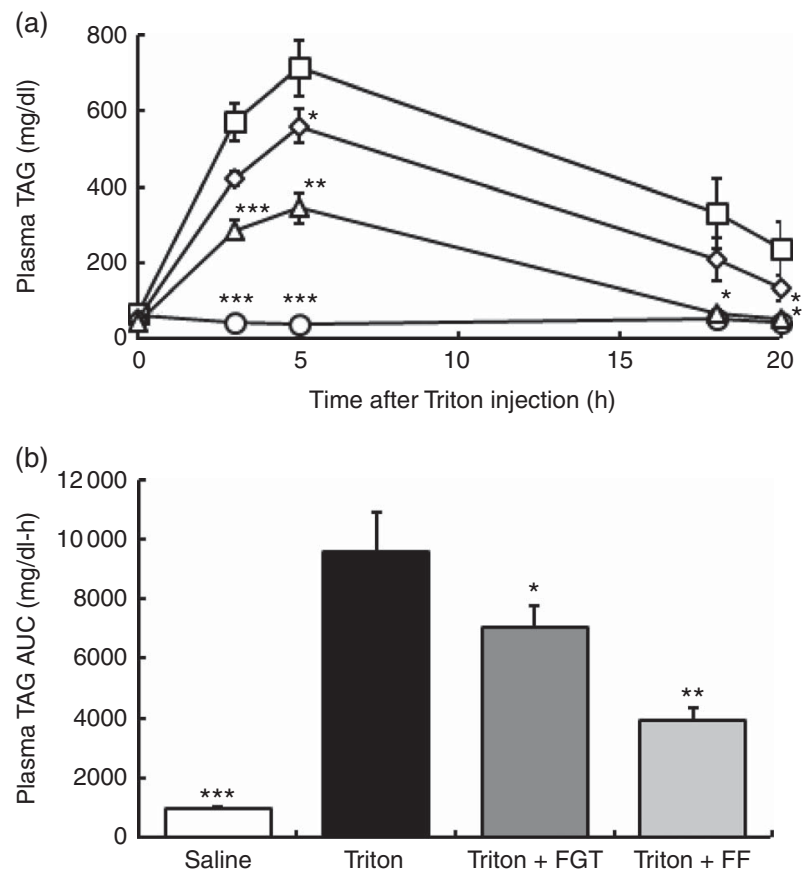

Fig. 1. Fermented green tea (FGT) alleviates acute hyperlipidaemia. SpragueDawley rats were administered saline $(-\mathrm{O}-)$, FGT (Triton + FGT ( $\prec)$; $500 \mathrm{mg} / \mathrm{kg}$ body weight) or fenofibrate (FF) (Triton + FF ( $-\triangle-) ; 65 \mathrm{mg} / \mathrm{kg}$ body weight) for $5 \mathrm{~d}$. After overnight fasting, Triton ( $\square-)(200 \mathrm{mg} / \mathrm{kg}$ body weight) was injected via the tail vein, and blood samples were collected at $0,3,5,18$ and $20 \mathrm{~h}$ after Triton administration. (a) Changes in plasma TAG levels are shown as line graphs, and (b) the AUC was calculated and presented as bar graphs ( $n$ 5/group). ${ }^{\star} P<0.05 \mathrm{v}$. Triton, ${ }^{\star \star} P<0.01 \mathrm{v}$. Triton, ${ }^{\star \star \star} ~ P<0.001 \mathrm{v}$. Triton. To convert TAG from $\mathrm{mg} / \mathrm{dl}$ to $\mathrm{mmol} / \mathrm{l}$, multiply by 0.0113 .
FGT on Western diet-fed hyperlipidaemic animals, Western diet-induced hyperlipidaemic hamsters were administered $\mathrm{FF}$ (100 mg/kg; as positive control) or FGT (200/400/600 mg/kg) for 12 weeks. Interestingly, FGT lowered plasma TAG levels in a dose-dependent manner (Fig. 2(B)). Thus, low-dose FGT likely requires a long time to exert its hypotriglyceridaemic effect, whereas a high-dose of FGT rapidly reduces plasma TAG levels.

\section{Fermented green tea inhibits pancreatic lipase activity}

We found that FGT blunted plasma TAG levels in hyperlipidaemic animal models (Fig. 1 and 2). However, it is unclear how FGT reduces plasma lipid levels. In order to identify potential hypolipidaemic mechanisms, we examined the promoter activity of PPAR $\alpha$, liver X receptor and forkhead box O, protein levels of LPL and activity of diacylglyceride acyltransferase; however, none of them was affected by FGT (online Supplementary Fig. S1). Dietary lipids are digested by pancreatic lipases and absorbed in the gut. Therefore, inhibition of pancreatic lipase would be a mechanism for the treatment of acquired hyperlipidaemia. To elucidate whether FGT-mediated hypotriglyceridaemic effect requires modulation of pancreatic lipase activity, we assessed pancreatic lipase inhibition assay using FGT. As shown in Fig. 3, FGT effectively and dose-dependently suppressed enzymatic activity of pancreatic lipase. Calculated from the experimental data, the $\mathrm{IC}_{50}$ of FGT on pancreatic lipase is $0.49 \mathrm{mg} / \mathrm{ml}$.
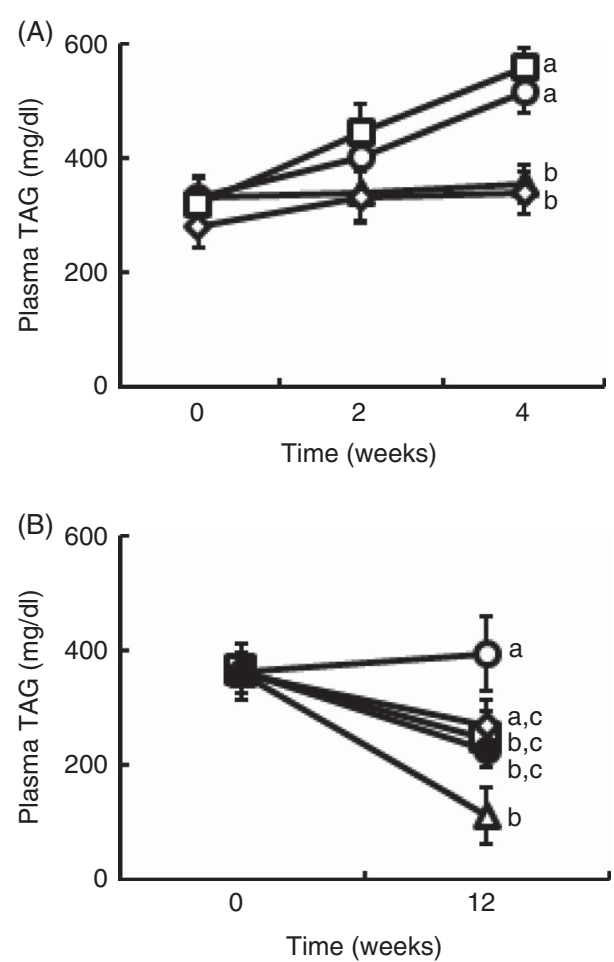

Fig. 2. Fermented green tea (FGT) exerts hypotriglyceridaemic effects. Hamsters fed a Western diet were administered vehicle, fenofibrate (FF, $\triangle-$ ) or FGT for 4 weeks (A) or 12 weeks (B). Plasma TAG levels are presented as line

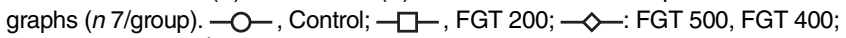
- - FGT 600 . $^{\text {a,b,c }}$ Mean values with unlike letters were significantly different $(P<0.05)$. To convert TAG from $\mathrm{mg} / \mathrm{dl}$ to $\mathrm{mmol} / \mathrm{l}$, multiply by 0.0113 . 
Fermented green tea augments energy expenditure, modulates the expressions of lipid metabolism-related genes and increases plasma serotonin levels

FGT inhibited pancreatic lipase activity and increased faecal lipid content (Fig. 3 and online Supplementary Fig. S2).

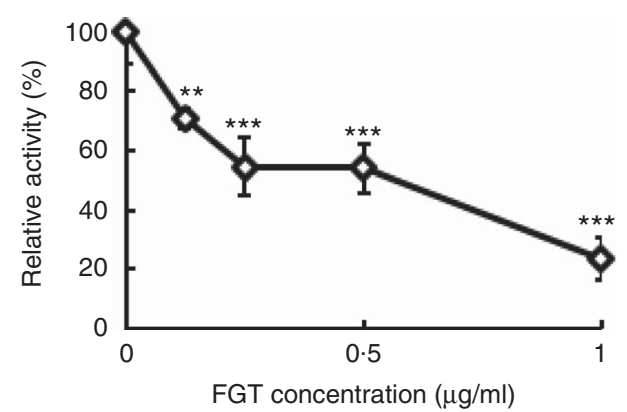

Fig. 3. Fermented green tea (FGT) inhibits pancreatic lipase activity. Enzymatic activity of pancreatic lipase is shown as line graph $(n 5)$. ${ }^{\star \star} P<0.01$ v. $0,{ }^{\star \star *} P<0.001$ v. 0.

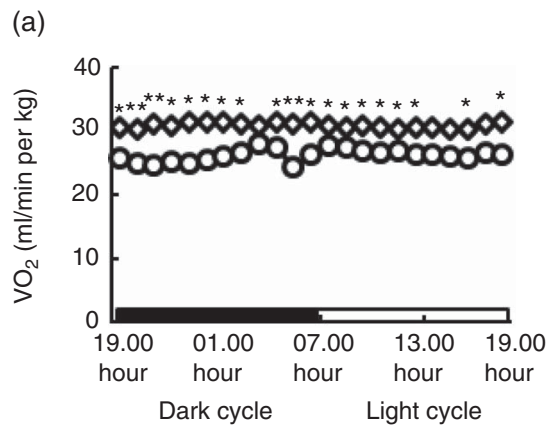

(b)

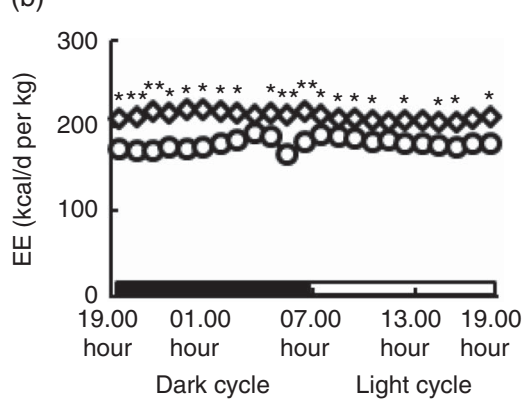

(c)

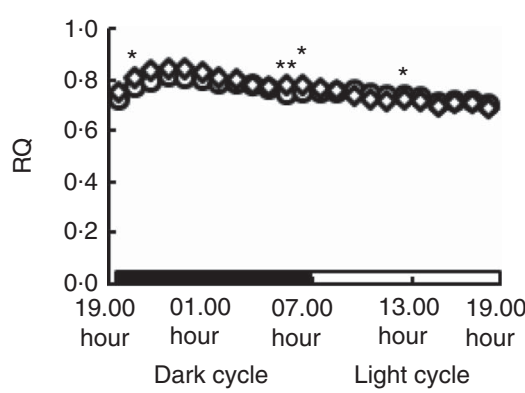

However, dietary lipids and pancreatic lipase are not the causal factors in Triton-induced acute hyperlipidaemia. This suggests that the hypotriglyceridaemic effect of FGT could be mediated by multiple mechanisms. Interestingly, we observed that FGT-administered animals were more active, compared with the vehicle group (data not shown), suggesting that FGT might affect energy expenditure. To determine FGT-mediated changes in energy expenditure, $\mathrm{C} 57 \mathrm{BL} / 6 \mathrm{~J}$ mice were fed a HFD with oral administration of FGT $(500 \mathrm{mg} / \mathrm{kg})$ for 2 weeks in an animal metabolic monitoring system. $\mathrm{VO}_{2}$ (Fig. 4(a)) and energy expenditure (Fig. 4(b)) were significantly elevated during the light and dark cycles in the FGT-fed mice, whereas the RQ was unchanged between FGT and vehicle groups (Fig. 4(c)). Thus, FGT appears to encourage energy expenditure without affecting energy source.

To further elucidate the effect of FGT on energy metabolism, we measured mRNA expressions of lipid metabolism-related genes (e.g. sterol regulatory element-binding protein-1c (SREBP1c), acetyl-CoA carboxylase (ACC), fatty acid synthase (FAS), stearoyl CoA desaturase-1 (SCD1), acyl-CoA oxidase
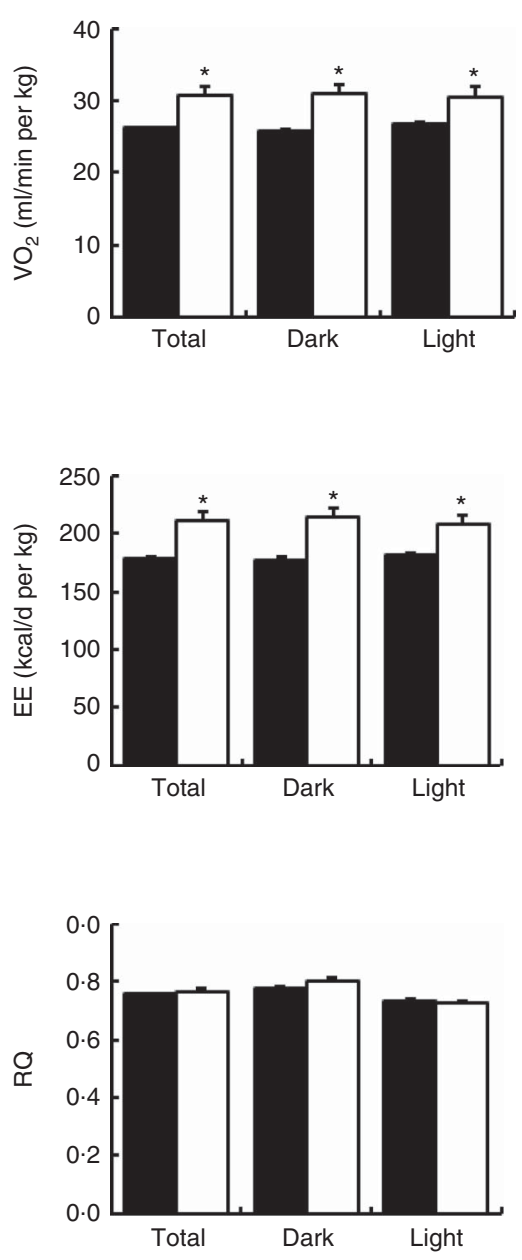

Fig. 4. Fermented green tea (FGT) increases energy expenditure. $V_{2}$ (a), energy expenditure (EE) (b) and $R Q$ (c) of $F G T$-treated mice were measured as described in the Methods and shown as bar and line graphs ( $\square$, control; $\square$, FGT). Indirect calorimetry was performed as described in the method. Dark cycle is from 19.00 to 07.00 hours and light cycle is from 07.00 to 19.00 hours, respectively ( $n$ 5/group). ${ }^{\star} P<0.05 \mathrm{v}$. control, ${ }^{\star *} P<0.01 \mathrm{v}$. control $(-\mathrm{O}-$, control; $-\diamond-$, FGT). To convert kcal to kJ, multiply by $4 \cdot 184$. 
(ACO), carnitine palmitate transferase-1 (CPT1), medium-chain acyl CoA dehydrogenase (mCAD) and PPAR $\alpha$ ) in WAT and the liver. Notably, expressions of lipogenic genes (SREBP1c, ACC, FAS and SCD1) were down-regulated (Fig. 5(a)), whereas expressions of fatty acid oxidation-related genes (ACO, CPT, mCAD and PPAR $\alpha$ ) remained up-regulated (Fig. 5(b)) in both tissues. These data imply that FGT might control the expressions of lipid metabolism-related genes to modulate circulating lipid levels. Surprisingly, plasma concentrations of serotonin, a neurotransmitter associated with energy expenditure and behaviour ${ }^{(40,41)}$, were significantly increased in FGT-administered mice (Fig. 6(a)), and the expressions of fatty acid oxidation genes were up-regulated by FGT as well as serotonin treatments in cultured adipocytes and myocytes, respectively (Fig. 6(b) and (c)). These results suggest that FGT stimulates lipid metabolism to increase energy expenditure by inducing serotonin.

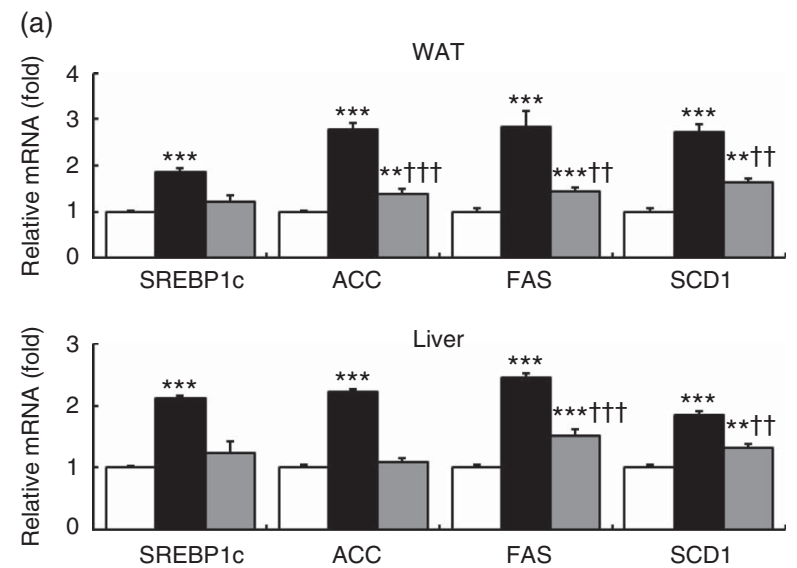

(b)
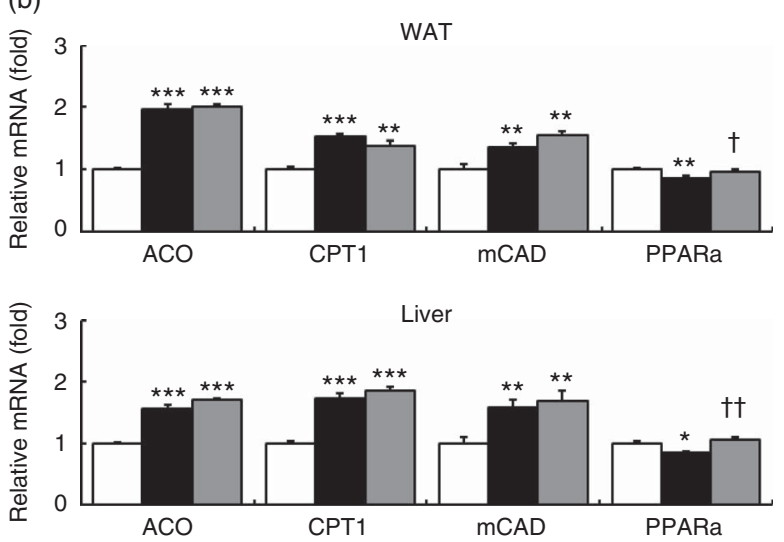

Fig. 5. Fermented green tea (FGT) modulates mRNA expressions of lipid metabolism-related genes in peripheral tissues. Gene expressions of sterol regulatory element-binding protein-1c (SREBP1c), acetyl-CoA carboxylase (ACC), fatty acid synthase (FAS), acyl-CoA oxidase (ACO) and carnitine palmitate transferase-1 (CPT) in adipose tissue (a) and liver (b) are shown as bar graphs (n 5/group). WAT, white adipose tissue; SCD1, stearoyl CoA desaturase-1, CPT1, carnitine palmitate transferase-1; mCAD, medium-chain acyl CoA dehydrogenase; $\square$, Control; $\square$, high-fat diet (HFD); $\square$, HFD + FGT. ${ }^{\star} P<0.05$ v. control, ${ }^{\star \star} P<0.01$ v. control, ${ }^{* \star *} P<0.001$ v. control, ${ }^{\dagger} P<0.05 \mathrm{v}$. HFD, ${ }^{\dagger \dagger} P<0.01$ v. HFD, ${ }^{\dagger \dagger \dagger} P<0.001$ v. HFD.
Fermented green tea changes the composition of gut microbiota in the hyperlipidaemic hamster model

It has been reported that metabolic disorders such as obesity and type 2 diabetes are closely related to alterations of the composition of gut microbiota, especially the Firmicutes phylum $^{(42-44)}$. We found that FGT reversed the changes in the composition of gut microbiota in diet-induced obese mice ${ }^{(35)}$. To determine whether FGT also altered the composition of gut
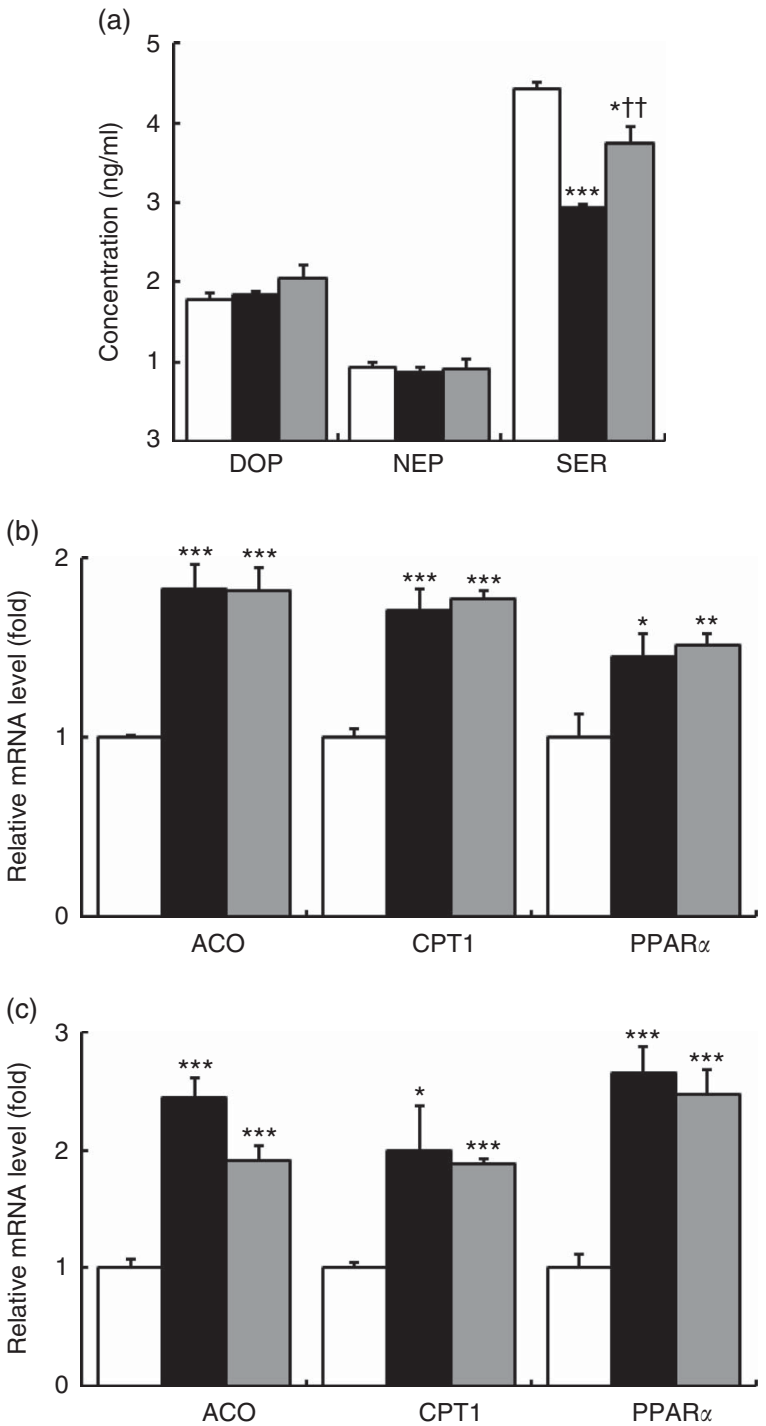

Fig. 6. Fermented green tea (FGT) increases plasma serotonin levels and fatty acid oxidation-related genes. (a)Plasma levels of neurotransmitters (dopamine (DOP), norepinephrine (NEP) and serotonin (SER)). $\square$, Normal diet group; $\square$, high-fat diet (HFD) group; $\square$, HFD with FGT. ${ }^{*} P<0.05 v$. control, ${ }^{\star \star \star} P<0.001$ v. control, ${ }^{\dagger \dagger} P<0.01$ v. HFD. Expressions of fatty acid oxidation genes in (b) 3T3-L1 adipocytes and (c) C2C12 myocytes. FGT $(500 \mu \mathrm{g} / \mathrm{ml})$ or serotonin (SER; $4 \mathrm{ng} / \mathrm{ml}$ ) treatment was performed for $24 \mathrm{~h}$. mRNA was isolated using Trizol $^{\mathrm{TM}}$ Reagent (Life Technologies). Each RNA sample $(2 \mu \mathrm{g})$ was subjected to complementary DNA (cDNA) synthesis using the RevertAid ${ }^{\mathrm{TM}}$ First Strand cDNA Synthesis Kit, and relative mRNA levels were determined by quantitative RT-PCR using the appropriate primers ( $n$ 4/group). ACO, acyl-CoA oxidase; CPT1, carnitine palmitate transferase-1; $\square$, not treated (negative control); $\square$, FGT; $\square$, SER. * $P<0.05$ v. negative control, ${ }^{\star *} P<0.01$ v. negative control, ${ }^{\star * \star} P<0.001$ v. negative control. 
(a)

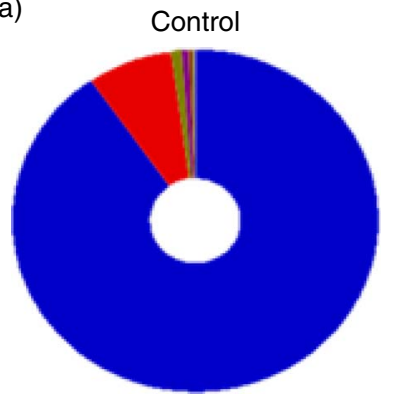

Phylum

Firmicutes(90.3\%)

Bacteroidetes $(7.56 \%)$

Proteobacteria $(0.95 \%)$

Actinobacteria $(0.59 \%)$

Verrucomicrobia $(0.4 \%)$

Cyanobacteria $(0.02 \%)$

TM7(0.1\%)

Tenericutes $(0.05 \%)$

Bacteria_uc $(0.02 \%)$

Elusimicrobia(0.02\%)

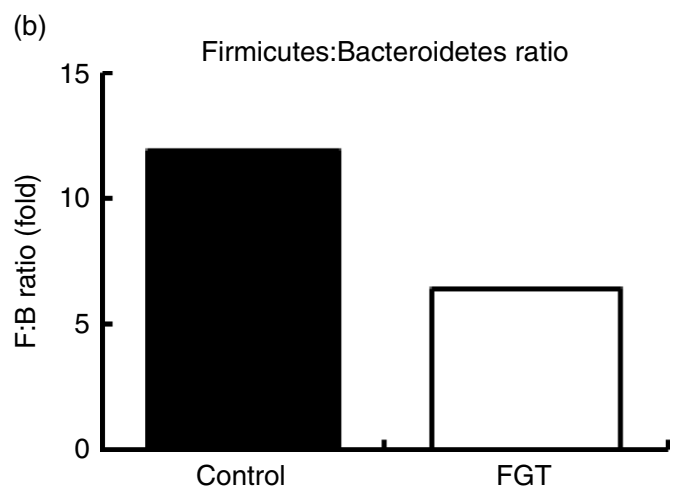

Fig. 7. Fermented green tea (FGT) alters the composition of gut microbiota in hamsters. Composition of gut microbiota (phylum level) (a) and ratio of Firmicutes:Bacteroidetes phylum (b) of the control and FGT $(500 \mathrm{mg} / \mathrm{kg}$ body weight)-administered hamsters. Stool samples from three hamsters were combined to analyse gut microbiota. For a colour figure, see the online version of the paper.

microbiota in Western diet-induced hyperlipidaemic hamsters, gut microbiota was analysed by pyrosequecing. Similar to previous results ${ }^{(35)}$, FGT slightly reduced the abundance of the Firmicutes phylum and enhanced the Bacteroidetes phylum, when compared with the vehicle group (Fig. 7(a)). The ratio of Firmicutes:Bacteroidetes was also reduced by FGT treatment (Fig. 7(b)). Collectively, it appears that FGT modulates gut microbiota by suppressing the prevalence of the Firmicutes phylum and facilitating the growth of Bacteroidetes, supporting a role for FGT in the development of metabolic disorders such as obesity and type 2 diabetes.

\section{Discussion}

In the present study, we show that FGT effectively rescued postprandial hypertriglyceridaemia and Western diet-induced hyperlipidaemia. We conducted in vivo experiments in different rodent models to confirm the effects of FGT in vivo. First, acute hypolipidaemic effects were examined in rats induced with postprandial lipaemia. Hamsters but not mice readily develop hypertriglyceridaemia on diets; thus, the long-term hypotriglyceridaemic effects of FGT were studied in hamsters. In addition, the metabolic rate and energy expenditure were measured in mice. Each animal model used in this study has been widely used for these experiments. The use of different animal models also shows that the hypolipidaemic effects of FGT are repeatedly found in different animal models, which confirms the validity of the hypolipidaemic effects of FGT.

Plasma TAG concentrations may be reduced by several biological mechanisms. Common therapeutics for hypertriglyceridaemia include the use fibrates or niacin ${ }^{(45)}$. Fibrates are ligand activators for the nuclear receptor PPAR $\alpha$. Activation of $\operatorname{PPAR} \alpha$ re-programmes gene expression in lipid metabolism, especially in the liver, thereby increasing fatty acid uptake and oxidation while suppressing VLDL secretion to lower plasma and hepatic TAG levels. Niacin binds and activates GPR109A, a niacin receptor, and suppresses the protein kinase A signalling pathway to lower adipocyte lipolysis, mobilisation of fatty acids to the liver and secretion of $\mathrm{VLDL}^{(46)}$. These effects result in the reduction of plasma TAG concentrations as well. In addition, activation of liver $\mathrm{X}$ receptor ${ }^{(47)}$ and inhibition of forkhead box $\mathrm{O}$ transcription factor ${ }^{(48)}$, LPL ${ }^{(49)}$ and diacylglycerol acyltransferase $^{(50)}$ are associated with the reduction in plasma TAG levels; however, none of those processes was affected by FGT in our activity screening experiments (online Supplementary Fig. S1).

Acute hypertriglyceridaemia in the postprandial state was ameliorated by inhibition of pancreatic lipase activity. Pancreatic lipase suppresses digestion and absorption of dietary lipids from meals; thus, inhibition of pancreatic lipase ameliorates postprandial lipaemia by FGT. Pancreatic lipase, a key-step enzyme in lipid digestion, catalyses the hydrolysis of dietary TAG into monoglyceride and fatty acids, so that dietary lipids are readily absorbed in the digestive tract ${ }^{(51)}$. Therefore, inhibition of pancreatic lipase activity serves as a primary target in the treatment of hyperlipidaemia. Indeed, orlistat, a pancreatic lipase inhibitor, is used to treat obesity by reducing excess energy intake. Interestingly, orlistat also has other biological effects including the reduction of blood pressure and reduction of the incidence of diabetes in human clinical trials with obese patients ${ }^{(52)}$. Whether these additional effects of orlistat are mediated through the suppression of lipid metabolism needs further evaluation. As orlistat, a pancreatic lipase inhibitor, controls hyperlipidaemia, it is feasible that FGT can potentially control hyperlipidaemia and related complications such as hypertension, type 2 diabetes and related CVD, as well as obesity.

In long-term feeding studies, plasma TAG levels were reduced in hamsters. FGT may reduce plasma TAG levels by increasing energy expenditure and serotonin secretion. We suggest that serotonin stimulates the consumption of stored lipid to lower plasma TAG levels, which could be due to induction of fatty acid oxidation gene expressions. Serotonin is a well-known neurotransmitter that is considered to be a 'happy hormone' because it is associated with feeding behaviour and $\operatorname{mood}^{(53)}$. Mood control has been identified as an important factor in reducing the progression of $\mathrm{CHD}$ and its associated mortality ${ }^{(8)}$. The FGT-associated increase in serotonin levels 
may be beneficial in alleviating this risk factor for CVD, which are often accompanied by hyperlipidaemia. Recently, serotonin has been associated with energy expenditure ${ }^{(40,41)}$. In this study, we demonstrated that FGT augments plasma serotonin levels (Fig. 6). Interestingly, we did not observe marked changes in the expression of tryptophan hydroxylase 1 (tph1) (online Supplementary Fig. S3), an enzyme that is involved in serotonin biosynthesis in the gut of FGT-treated mice. This observation suggests that the effects of FGT on serotonin metabolism involve a different pathway of regulation of serotonin metabolism by FGT, which requires further studies. It is possible that FGT boosts whole-body energy expenditure to reduce circulating lipid levels by regulating serotonin, at least in part. By augmenting serotonin metabolism, FGT is also expected to modulate happiness and reduce the development of CVD, both of which are thought to be influenced by lipid metabolism and mood. Enhanced energy expenditure reflects a huge consumption of energy, which accompanies a robust increase in lipid catabolism to supply ATP demand. We observed that FGT administration suppressed lipogenic gene expression while enhancing catalytic gene expression in peripheral tissues (Fig. 5), implying that the pattern of mRNA expression of lipid metabolism-related genes shifted favourably from lipogenic to lipolytic following FGT treatment. By combining two mechanisms, inhibition of pancreatic lipase and induction of serotonin secretion, FGT may effectively reduce plasma TAG levels.

In addition, it is possible that FGT compounds may modulate key metabolic regulators including AMPK, silent mating type information regulation 2 homolog 1 (Sirt1) and PGC1 $\alpha^{(54-56)}$. In a previous study, the amount of gallic acid robustly increased during green tea fermentation ${ }^{(57)}$. Recently, gallic acid has been reported to exhibit anti-obesity and anti-diabetic properties through the activation of AMPK, Sirt1 and PGC1 $\alpha^{(58)}$. EGCG, a major component of green tea, also modulates energy metabolism through AMPK activation ${ }^{(59-61)}$. Although the content of EGCG in FGT is much lower than that of green tea, the catechins and increased gallates (possibly due to metabolism of catechin gallates) are able to mediate the hypotriglyceridaemic effects of FGT. Furthermore, there are more active compounds that are effective in modulating lipid metabolism in processed green teas. For instance, theaflavins from black tea reduce cholesterol incorporation into micelles ${ }^{(62)}$, thereby reducing cholesterol uptake. Although we have not yet identified active compounds for key metabolic regulators, we are presently attempting to identify the major polyphenolic compounds in FGT by utilising various biochemical analytical methods. Further research is required to identify the active components and to evaluate the detailed mechanism underlying FGT-mediated hypotriglyceridaemic effects.

Changes in the gut microbiota is closely correlated with the development and treatment of lipid metabolism-related disorders including obesity and type 2 diabetes ${ }^{(63,64)}$. In the analysis of microbiota changes, the ratio of Firmicutes: Bacteroidetes has been suggested as an informative biomarker for metabolic disorders, as this ratio is closely associated with the development of obesity ${ }^{(65)}$ and type 2 diabetes ${ }^{(63)}$. We previously reported that FGT reduced the Firmicutes:
Bacteroidetes ratio in mouse gut microbiota, and the present study confirms the previous findings in hamster microbiota ${ }^{(35)}$. In the present study, changes in microbiota were associated with complex metabolic alterations including reduced TAG levels and body weight; thus, it is not possible to characterise microbiota changes specific to hypotriglycaeridaemic effects. However the Firmicutes:Bacteroidetes ratio in hamsters was significantly reduced, which confirmed our previous findings. It has been suggested that the phylum Firmicutes predominates the gut microbiota of obese mice ${ }^{(42)}$; thus, the host likely receives more energy content with increasing Firmicutes levels in the gut. Therefore, FGT-induced alteration of the composition of the gut microbiota (reduced Firmicutes) contributed to the reduction in energy intake in the absence of a change in food intake, thereby reducing body weight gain and fat mass increase, at least in part.

In the analysis of gut microbiota, the most abundant genera was Allobaculum, which was increased in the FGT group ( 45.7 and $53.6 \%$ in control and FGT, respectively). Allobaculum was shown to be enriched after exercise in rats ${ }^{(66)}$, augmented when supplemented with grain sorghum lipid extract in hamsters ${ }^{(67)}$ and increased with improved metabolic parameters in obese and insulin-resistant rats after berberine feeding ${ }^{(68)}$. In addition, Ruminococcus was reduced in hamsters fed FGT (8.3 and 5.7\% in control and FGT, respectively). Ruminococcus has been found to be more abundant in obese subjects than in non-obese subjects ${ }^{(69)}$. These changes may be associated with the hypotriglyceridaemic effects of FGT, and further studies will be performed on this issue in the future.

In conclusion, FGT inhibits pancreatic lipase activity and induces serotonin secretion to modulate lipid metabolism and reduces hyperlipidaemia in animal models. We propose that FGT may be a novel hypotriglyceridaemic agent for the treatment of lipid dysregulation and related complications.

\section{Acknowledgements}

The authors appreciate Insik Lee, Kiyeop Park and Jinsang Jung (Aestra Co.) for care and assistance with the animal experiments with SD rats. The authors also appreciate Chae Wook Kim for measuring the physical activity of FGT-administered mice. All the authors have read the journal's policy and disclose the following conflicts. Authors H. W. J., S. K., J. K., J. H. L., K. J. and S. S. S. are employees of Amorepacific Corporation. J. K. C. is an employee of Aeustra Corporation. A patent application for the improvement of lipid dysregulation titled 'Composition comprising fermented tea extracts for reducing lipid level' was submitted on and is currently undergoing revision (submission number 1020100125468 for Republic of Korea and PCT/ KR2011/009502 for patent cooperation treaty, respectively). There is one related product on the market in South Korea (S'Lite Slimmer DX). However, this does not alter our adherence to the policies of the Food Research International on data and material sharing.

This work was supported by the National Research Foundation of Korea (NRF) grant funded by the Korea government (MSIP) (no. NRF-2016R1A2A2A05005483) and Amorepacific 
Research Institute (Q1304251). NRF and Amorepacific Research Institute had no role in the design.

D.-B. S., H. W. J., Y.-J. K. and J. K. C. performed animal experiments and data analysis. S. K., J. K., J. H. L and K. J. measured serotonin levels. S.-J. L. and S. S. K. organised and designed the experiments. S.-J. L., D.-B. S., H. W. J. and Y.-J. K. wrote the manuscript.

The authors declare that there are no conflicts of interest.

\section{Supplementary material}

For supplementary material/s referred to in this article, please visit https://doi.org/10.1017/S0007114516004621

\section{References}

1. Kopelman PG (2000) Obesity as a medical problem. Nature 404, 635-643.

2. Williams KJ (2008) Molecular processes that handle - and mishandle - dietary lipids. J Clin Invest 118, 3247-3259.

3. Cheung O \& Sanyal AJ (2008) Abnormalities of lipid metabolism in nonalcoholic fatty liver disease. Semin Liver Dis $\mathbf{2 8}$, 351-359.

4. Goodpaster BH \& Kelley DE (2002) Skeletal muscle triglyceride: marker or mediator of obesity-induced insulin resistance in type 2 diabetes mellitus? Curr Diab Rep 2, 216-222.

5. Raal FJ (2009) Pathogenesis and management of the dyslipidemia of the metabolic syndrome. Metab Syndr Relat Disord 7, 83-88.

6. Roden M, Price TB, Perseghin G, et al. (1996) Mechanism of free fatty acid-induced insulin resistance in humans. $J$ Clin Invest 97, 2859-2865.

7. Zraika S, Dunlop M, Proietto J, et al. (2002) Effects of free fatty acids on insulin secretion in obesity. Obes Rev 3, 103-112.

8. Nelson RH (2013) Hyperlipidemia as a risk factor for cardiovascular disease. Prim Care 40, 195-211.

9. Athyros VG \& Wierzbicki AS (2013) Statin-fibrate combination therapy is safe and effective in normalizing lipid profile and in keeping cardiovascular event rates low. Curr Med Res Opin 30, $57-58$.

10. Fazio S (2008) Management of mixed dyslipidemia in patients with or at risk for cardiovascular disease: a role for combination fibrate therapy. Clin Ther 30, 294-306.

11. Fedele D, Tiengo A, Nosadini R, et al. (1976) Hypolipidemic effects of metformin in hyperprebetalipoproteinemia. Diabete Metab 2, 127-133.

12. Jensen GB, Hilden J, Als-Nielsen B, et al. (2010) Statin treatment prevents increased cardiovascular and all-cause mortality associated with clarithromycin in patients with stable coronary heart disease. J Cardiovasc Pharmacol 55, 123-128.

13. Brusq JM, Ancellin N, Grondin P, et al. (2006) Inhibition of lipid synthesis through activation of AMP kinase: an additional mechanism for the hypolipidemic effects of berberine. J Lipid Res 47, 1281-1288.

14. Kim WS, Lee YS, Cha SH, et al. (2009) Berberine improves lipid dysregulation in obesity by controlling central and peripheral AMPK activity. Am J Physiol Endocrinol Metab 296, E812-E819.

15. Lee YS, Kim WS, Kim KH, et al. (2006) Berberine, a natural plant product, activates AMP-activated protein kinase with beneficial metabolic effects in diabetic and insulinresistant states. Diabetes 55, 2256-2264.

16. Alshatwi AA, Al Obaaid MA, Al Sedairy SA, et al. (2010) Black and green tea improves lipid profile and lipid peroxidation parameters in Wistar rats fed a high-cholesterol diet. J Physiol Biochem 67, 95-104.

17. Amanolahi F \& Rakhshande H (2013) Effects of ethanolic extract of green tea on decreasing the level of lipid profile in rat. Avicenna J Phytomed 3, 98-105.

18. Cao ZH, Gu DH, Lin QY, et al. (2010) Effect of pu-erh tea on body fat and lipid profiles in rats with diet-induced obesity. Phytother Res 25, 234-238.

19. Haidari F, Shahi MM, Zarei M, et al. (2012) Effect of green tea extract on body weight, serum glucose and lipid profile in streptozotocin-induced diabetic rats. A dose response study. Saudi Med J 33, 128-133.

20. Li RW, Douglas TD, Maiyoh GK, et al. (2006) Green tea leaf extract improves lipid and glucose homeostasis in a fructosefed insulin-resistant hamster model. J Ethnopharmacol 104, 24-31.

21. Serisier S, Leray V, Poudroux W, et al. (2008) Effects of green tea on insulin sensitivity, lipid profile and expression of PPARalpha and PPARgamma and their target genes in obese dogs. Br J Nutr 99, 1208-1216.

22. Skrzydlewska E, Ostrowska J, Farbiszewski R, et al. (2002) Protective effect of green tea against lipid peroxidation in the rat liver, blood serum and the brain. Phytomedicine $9,232-238$.

23. Toyoda-Ono Y, Yoshimura M, Nakai M, et al. (2007) Suppression of postprandial hypertriglyceridemia in rats and mice by oolong tea polymerized polyphenols. Biosci Biotechnol Biochem 71, 971-976.

24. Vinson JA \& Dabbagh YA (1998) Effect of green and black tea supplementation on lipids, lipid oxidation and fibrinogen in the hamster: mechanisms for the epidemiological benefits of tea drinking. FEBS Lett 433, 44-46.

25. Yang M, Wang C \& Chen H (2001) Green, oolong and black tea extracts modulate lipid metabolism in hyperlipidemia rats fed high-sucrose diet. J Nutr Biochem 12, 14-20.

26. Lee SJ \& Jia Y (2015) The effect of bioactive compounds in tea on lipid metabolism and obesity through regulation of peroxisome proliferator-activated receptors. Curr Opin Lipidol 26, 3-9.

27. Walkowiak J, Bajerska J, Kargulewicz A, et al. (2013) Single dose of green tea extract decreases lipid digestion and absorption from a test meal in humans. Acta Biochim Pol 60 , 481-483.

28. Basu A, Sanchez K, Leyva MJ, et al. (2010) Green tea supplementation affects body weight, lipids, and lipid peroxidation in obese subjects with metabolic syndrome. J Am Coll Nutr 29, 31-40.

29. Erba D, Riso P, Bordoni A, et al. (2005) Effectiveness of moderate green tea consumption on antioxidative status and plasma lipid profile in humans. J Nutr Biochem 16, 144-149.

30. Davies MJ, Judd JT, Baer DJ, et al. (2003) Black tea consumption reduces total and LDL cholesterol in mildly hypercholesterolemic adults. J Nutr 133, 3298S-3302S.

31. Fujita H \& Yamagami $T$ (2008) Antihypercholesterolemic effect of Chinese black tea extract in human subjects with borderline hypercholesterolemia. Nutr Res 28, 450-456.

32. Zhao Y, Asimi S, Wu K, et al. (2015) Black tea consumption and serum cholesterol concentration: systematic review and meta-analysis of randomized controlled trials. Clin Nutr $\mathbf{3 4}$, 612-619.

33. Singh DK, Banerjee S \& Porter TD (2009) Green and black tea extracts inhibit HMG-CoA reductase and activate AMP kinase to decrease cholesterol synthesis in hepatoma cells. $J$ Nutr Biochem 20, 816-822.

34. Hsu TF, Kusumoto A, Abe K, et al. (2006) Polyphenolenriched oolong tea increases fecal lipid excretion. Eur J Clin Nutr 60, 1330-1336. 
35. Seo DB, Jeong HW, Cho D, et al. (2015) Fermented green tea extract alleviates obesity and related complications and alters gut microbiota composition in diet-induced obese mice. J Med Food 18, 549-556.

36. Nakai M, Fukui Y, Asami S, et al. (2005) Inhibitory effects of oolong tea polyphenols on pancreatic lipase in vitro.J Agric Food Chem 53, 4593-4598.

37. Chun J, Kim KY, Lee JH, et al. (2010) The analysis of oral microbial communities of wild-type and toll-like receptor 2-deficient mice using a 454 GS FLX Titanium pyrosequencer. BMC Microbiol 10, 101.

38. Kim OS, Cho YJ, Lee K, et al. (2012) Introducing EzTaxon-e: a prokaryotic $16 \mathrm{~S}$ rRNA gene sequence database with phylotypes that represent uncultured species. Int J Syst Evol Microbiol 62, 716-721.

39. Hoang MH, Jia Y, Jun HJ, et al. (2012) Taurine is a liver X receptor-alpha ligand and activates transcription of key genes in the reverse cholesterol transport without inducing hepatic lipogenesis. Mol Nutr Food Res 56, 900-911.

40. Watanabe H, Rose MT \& Aso H (2011) Role of peripheral serotonin in glucose and lipid metabolism. Curr Opin Lipidol 22, 186-191.

41. Watanabe H, Akasaka D, Ogasawara H, et al. (2010) Peripheral serotonin enhances lipid metabolism by accelerating bile acid turnover. Endocrinology 151, 4776-4786.

42. Ley RE, Backhed F, Turnbaugh $\mathrm{P}$, et al. (2005) Obesity alters gut microbial ecology. Proc Natl Acad Sci U S A 102, 11070-11075.

43. DiBaise JK, Zhang H, Crowell MD, et al. (2008) Gut microbiota and its possible relationship with obesity. Mayo Clin Pro $\mathbf{8 3}$, 460-469.

44. Qiao Y, Sun J, Xia S, et al. (2014) Effects of resveratrol on gut microbiota and fat storage in a mouse model with high-fat-induced obesity. Food Funct 5, 1241-1249.

45. Vrablik M \& Ceska R (2015) Treatment of hypertriglyceridemia: a review of current options. Physiol Res 64, Suppl. 3, S331-S340.

46. Digby JE, Ruparelia N \& Choudhury RP (2012) Niacin in cardiovascular disease: recent preclinical and clinical developments. Arterioscler Thromb Vasc Biol 32, 582-588.

47. Briand O, Touche V, Colin S, et al. (2016) Liver X receptor regulates triglyceride absorption through intestinal down-regulation of scavenger receptor class B, type 1 . Gastroenterology 150, 650-658.

48. Zhang K, Li L, Qi Y, et al. (2012) Hepatic suppression of Foxo1 and Foxo3 causes hypoglycemia and hyperlipidemia in mice. Endocrinology 153, 631-646.

49. Geldenhuys WJ, Lin L, Darvesh AS, et al. (2016) Emerging strategies of targeting lipoprotein lipase for metabolic and cardiovascular diseases. Drug Discov Today. (epublication ahead of print version 19 October 2016).

50. Naik R, Obiang-Obounou BW, Kim M, et al. (2014) Therapeutic strategies for metabolic diseases: small-molecule diacylglycerol acyltransferase (DGAT) inhibitors. Chem Med Chem 9, 2410-2424.

51. Lunagariya NA, Patel NK, Jagtap SC, et al. (2014) Inhibitors of pancreatic lipase: state of the art and clinical perspectives. EXCLI J 13, 897-921.

52. Torgerson JS, Hauptman J, Boldrin MN, et al. (2004) XENical in the prevention of diabetes in obese subjects (XENDOS) study: a randomized study of orlistat as an adjunct to lifestyle changes for the prevention of type 2 diabetes in obese patients. Diabetes Care 27, 155-161.

53. Young SN (2007) How to increase serotonin in the human brain without drugs. J Psychiatry Neurosci 32, 394-399.

54. Finck BN \& Kelly DP (2006) PGC-1 coactivators: inducible regulators of energy metabolism in health and disease. J Clin Invest 116, 615-622.

55. Hardie DG (2007) AMP-activated/SNF1 protein kinases: conserved guardians of cellular energy. Nat Rev Mol Cell Biol 8, 774-785.

56. Li X (2013) SIRT1 and energy metabolism. Acta Biochim Biophys Sin (Shanghai) 45, 51-60.

57. Lee JE, Lee BJ, Chung JO, et al. (2011) ${ }^{1} \mathrm{H}$ NMR-based metabolomic characterization during green tea (Camellia sinensis) fermentation. Food Res Int 44, 597-604.

58. Doan KV, Ko CM, Kinyua AW, et al. (2015) Gallic acid regulates body weight and glucose homeostasis through AMPK activation. Endocrinology 156, 157-168.

59. Cai EP \& Lin JK (2009) Epigallocatechin gallate (EGCG) and rutin suppress the glucotoxicity through activating IRS2 and AMPK signaling in rat pancreatic beta cells. J Agric Food Chem 57, 9817-9827.

60. Liu HW, Chan YC, Wang MF, et al. (2015) Dietary (-)-epigallocatechin-3-gallate supplementation counteracts aging-associated skeletal muscle insulin resistance and fatty liver in senescence-accelerated mouse. J Agric Food Chem $\mathbf{6 3}$, $8407-8417$

61. Xiao N, Mei F, Sun Y, et al. (2014) Quercetin, luteolin, and epigallocatechin gallate promote glucose disposal in adipocytes with regulation of AMP-activated kinase and/or sirtuin 1 activity. Planta Med 80, 993-1000.

62. Vermeer MA, Mulder TP \& Molhuizen HO (2008) Theaflavins from black tea, especially theaflavin-3-gallate, reduce the incorporation of cholesterol into mixed micelles. J Agric Food Chem 56, 12031-12036.

63. Qin J, Li Y, Cai Z, et al. (2012) A metagenome-wide association study of gut microbiota in type 2 diabetes. Nature $\mathbf{4 9 0}$, $55-60$.

64. Sanz Y, Santacruz A \& Gauffin P (2010) Gut microbiota in obesity and metabolic disorders. Proc Nutr Soc 69, 434-441.

65. Ley RE, Turnbaugh PJ, Klein S, et al. (2006) Microbial ecology: human gut microbes associated with obesity. Nature $\mathbf{4 4 4}$, 1022-1023.

66. Petriz BA, Castro AP, Almeida JA, et al. (2014) Exercise induction of gut microbiota modifications in obese, non-obese and hypertensive rats. BMC Genomics 15, 511.

67. An C, Kuda T, Yazaki T, et al. (2013) FLX pyrosequencing analysis of the effects of the brown-algal fermentable polysaccharides alginate and laminaran on rat cecal microbiotas. Appl Environ Microbiol 79, 860-866.

68. Zhang X, Zhao Y, Zhang M, et al. (2012) Structural changes of gut microbiota during berberine-mediated prevention of obesity and insulin resistance in high-fat diet-fed rats. PLOS ONE 7, e42529.

69. Kasai C, Sugimoto K, Moritani I, et al. (2015) Comparison of the gut microbiota composition between obese and nonobese individuals in a Japanese population, as analyzed by terminal restriction fragment length polymorphism and nextgeneration sequencing. BMC Gastroenterol 15, 100. 